\title{
Photoelectron backscattering effects in photoemission from CsI into gas media
}

\author{
A. Di Mauro, E. Nappi, F. Posa \\ INFN Sez. Bari and Politecnico, 70126 Bari, Italy \\ A. Breskin, A. Buzulutskov, R. Chechik \\ Department of Particle Physics, Weizmann Institute of Science, \\ 76100 Rehovot, Israel \\ S.F. Biagi \\ Department of Physics, University of Liverpool, United Kingdom \\ G. Paic \\ SUBATECH, Nantes, France \\ F. Piuz \\ CERN, Geneva, Switzerland
}

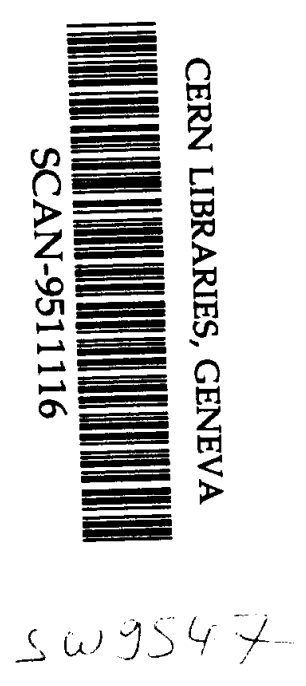

\begin{abstract}
The photoemission from solid surfaces into gas is important in view of the application of solid photocathodes in fast-RICH devices. The photoemission from CsI into gas has been investigated in $\mathrm{He}-$, Ar- and $\mathrm{CH}_{4}$-based gas mixtures as a function of the electric field at the photocathode surface. Measurements were made both in laboratory, with a UV source, and in a beam with a RICH detector. The results are interpreted in terms of current models of electron transport in gas. The electron collectioli efficiency, below gas ionization threshold, is reduced by backscattering. This phenomenon is particularly important in He-based gas mixtures. In $\mathrm{CH}_{4}$ and $\mathrm{Ar} / \mathrm{CH}_{4}$ mixtures the backscattering is very low. At high electric field, under charge multiplication, a full collection efficiency, similar to that in vacuum, is obtained in all gases investigated. We discuss the parameters governing the choice of the gas mixture in this kind of photon detectors.
\end{abstract}

Presented by A. Di Mauro at the RICH'95 Workshop, Uppsala, Sweden, June 12-16 1995. 


\section{Introduction.}

In recent years there has been a growing interest in UV-photon detectors combining solid photocathodes, in particular CsI, with gaseous photoelectron multipliers [1]. The importance of surface photoemission, as opposed to conversion in gas, is the fast timing and improved localization. The detectors have a reduced sensitivity to ionizing background and therefore a very low occupancy. This is of great importance in high multiplicity environment.

The CERN RD26 Collaboration is currently investigating the feasibility of operating large area CsI-based RICH counters. Potential applications foreseen are for example at the LHC-ALICE and the GSI-HADES experiments. Systematic investigations on UV-detectors combining large area CsI-coated pad electrodes and atmospheric pressure MWPCs have shown very satisfactory results, in terms of UV-photon detection efficiency and localization properties [2,3]. Since the particle identification capability of RICH devices is closely related to the number of photons detected per Cherenkov ring, efforts have been made on the one hand to optimize the photocathode production technique and on the other hand to understand the photoemission process $[4,5]$.

It has been noticed in the past that, unlike the case of emission from a solid surface into vacuum, the collection efficiency of photoelectrons emitted into gas depends on the gas composition and pressure [6-8]. Indeed, it is affected by the elastic backscattering of electrons on gas molecules. In noble gases and in some hydrocarbons, this effect considerably reduces the detector quantum efficiency. Recently, it has been recognized that the backscattering process is noticeably reduced under high electric fields at the photocathode vicinity, namely, under gas multiplication [9]. It has been demonstrated that, in such conditions, the photoelectron collection efficiency in a variety of gases can reach that of vacuum $[9,10]$. Indeed, the large mass difference between elastically scattered electrons and gas molecules determines a wide range of scattering angles, with a small energy transfer per collision. Below ionization threshold the backscattering effect is therefore more pronounced, in particular in noble gases, where the elastic cross-section largely exceeds the inelastic one, because of the lack of vibrational and rotational levels. Above the ionization threshold, the majority of collisions involves large energy losses; the electrons produced in these ionizing collisions are of low energy and they follow the accelerating electric field without backscattering.

In the present article, the electron transport process in the gas under electric fields has been modelled, taking into account recently calculated collisional cross-sections. We present here a comparison between experimental [9-10] and simulation results, demonstrating the validity of the backscattering hypothesis. We discuss also the relevant role of the choice of gas in UV-photon detectors for RICH. 


\section{Summary of experimental procedures and re- sults.}

A detailed presentation of experimental set-up, measurement procedures and results is given in $[9,10]$. The dependence of measured CsI photoemission into gas media on the gas nature and pressure and on the electric field at the photocathode vicinity was studied both in laboratory, with a UV source, and in a beam, with a RICH detector. He-, Ar- and $\mathrm{CH}_{4}$-based mixtures, with some quencher $\left(\mathrm{CH}_{4}\right.$ or $\left.\mathrm{i}-\mathrm{C}_{4} \mathrm{H}_{10}\right)$, were studied in the pressure range of 200-800 Torr.

In the UV tests two different experimental procedures were used: with the single pulse counting technique, the intensity of a pulsed discharge $\mathrm{H}_{2}$ lamp was adjusted to provide single photons and the photoelectrons extracted from a CsI photocathode were detected and counted by a detector made of a 2-stage PPAC (parallel plate avalanche chamber) coupled to a MWPC; with the $D C$ current recording technique, the photocurrent induced by a continuous flux $\mathrm{Hg}(\mathrm{Ar}$ ) lamp (emission peak at 185 $\mathrm{nm}$ ) was measured directly on the CsI photocathode. The single pulse counting technique allows to vary the field at the vicinity of the CsI surface while maintaining the pulse detection efficiency, so that we can compare the photoelectron collection efficiency measured at low electric fields, under "charge collection mode", to the one observed in high electric fields, under "charge multiplication mode". In the DC current recording, an absolute evaluation of the photoelectron collection efficiency is obtained by the ratio of the photocurrents measured in gas to that measured in vacuum, where the backscattering effect is absent.

In $\mathrm{He} / \mathrm{CH}_{4}(95 / 5)$ and $\mathrm{He} / \mathrm{i}-\mathrm{C}_{4} \mathrm{H}_{10}(95 / 5)$ the measured photoemission by pulse counting in the charge collection plateau is about $60 \%$ less than that in the charge multiplication plateau (fig. 1a,b). The photocurrent measured in DC mode in the same gas mixtures, below the multiplication threshold, is reduced by the same factor with respect to vacuum (fig. 2). Therefore, we deduce that at high electric fields, above a gas amplification of 100 , the same photoyield as in vacuum is obtained.

In $\mathrm{CH}_{4}$ and $\mathrm{CH}_{4} / \mathrm{i}-\mathrm{C}_{4} \mathrm{H}_{10}(95 / 5)$, in the charge collection mode, the photoelectron collection efficiency, derived from the pulse counting, is about $90 \%$ of the value in the multiplication mode (figs. 4 and 5 in [10] and fig 1c). Also in these gases, the gas-to-vacuum photocurrent ratio ( $\sim 0.9$ below multiplication) measured in the DC current technique (Fig. 3) is almost equal to the reduction factor estimated with the single pulse counting technique. These experimental evidences support the assumption that gas effects on the photoelectron collection efficiency are suppressed under charge multiplication $[9,10]$.

Ar-based mixtures were studied only with the DC current recording technique. The photocurrent ratio at the end of the charge collection plateau is $\sim 0.9 \mathrm{in} \mathrm{Ar} / \mathrm{CH}_{4}$ $(80 / 20)$ and $\sim 0.7$ in $\mathrm{Ar} / \mathrm{i}-\mathrm{C}_{4} \mathrm{H}_{10}(80 / 20)$ (fig. 3 in [10]). In $\mathrm{Ar} / \mathrm{CH}_{4}(50 / 50)$ the photoelectron collection efficiency approaches that in pure $\mathrm{CH}_{4}$ (fig. 4).

A good verification of the laboratory measurements comes from a RICH detector operated with some of the gas mixtures used in the laboratory UV test (Table 1). 
In the case of RICH, the photoelectron collection efficiency is estimated by the ratio $\mathrm{R}_{p e}$ of the average number of photoelectrons detected per event in the various gas mixtures to that in pure $\mathrm{CH}_{4}$. Indeed the best performances were obtained with this gas, used for normal operation of the RD26 RICH prototype, as foreseen by the laboratory measurements. For comparison the same ratio $R_{i}$ is calculated for the collection efficiencies obtained in laboratory and shown in the table. The average value of $E / p$ at the photocaihode surface of the MWPC of the UV-RICH detector, corresponding to the voltage set on its electrodes, was evaluated with the GARFIELD program.

\section{Monte Carlo simulation scheme of photoelec- tron transport in gas.}

The Monte Carlo program was implemented in order to reproduce the results obtained with the current recording technique, simulating the photoelectron transport process from the photocathode to the anode, in the same gas mixtures and $E / p$ values used in the measurements. Input parameters are: mixture composition, electric field, gas pressure and temperature and the maximum electron energy $\left(\epsilon_{\max }\right) . \epsilon_{\max }$ is the only adjustable parameter; its value depends on the electron energy distribution function in the gas mixture and it should be large enough to avoid significant cuts of the high energy tail.

In the first stage, single photoelectron emission energies are generated from a gaussian energy distribution with a mean of $0.26 \mathrm{eV}$ and a FWHM of $0.3 \mathrm{eV}$; these values are extrapolated from the curves in $[11,12]$ for $6.7 \mathrm{eV}$, corresponding to the average energy of photons emitted by the $\mathrm{Hg}(\mathrm{Ar})$ lamp. Then the emission angle is generated randomly in the interval $\left[0 . \theta_{n, a x}\right]$ defining the so called escape cone [13], where

$$
\theta_{\text {max }}=\arccos \sqrt{\frac{E_{a}}{E_{\text {start }}}}
$$

with $E_{a}=0.1 \mathrm{eV}$, the Csl electron affinity, and $E_{\text {start }}$ the randomly generated photoelectron emission energy.

The electron path and the physical variables are sampled at random time intervals in accordance with the null collision method that provides an efficient stochastic choice of the sampling time $t_{c}[14] . t_{c}$ is defined by the relation:

$$
\therefore=\frac{\ln (1-r n d m)}{\varkappa^{\prime}}
$$

where $\nu^{\prime}$ is a value of the collision frequency. larger than the maximum real collision frequency and $r n d m$ is a random number uniformly distributed in $[0,1]$; therefore a number $\left(\nu^{\prime}-\nu(\epsilon)\right)$ of collision per unit time should result null, leaving unchanged the electron energy and position. The outcome of each collision is determined by comparison of random numbers to the collision frequencies $\nu(\epsilon)$, calculated according 
to the formula [15]:

$$
\nu(\epsilon)=\sqrt{\frac{2 \epsilon}{m_{e}}} \sum_{k=1}^{N} n_{k}\left\{\sigma_{k}(\epsilon)+\sum_{i}\left(\sigma_{k}\right)_{i}(\epsilon)\right\},
$$

where $\sqrt{\frac{2 \epsilon}{m_{e}}}$ is the electron velocity corresponding to the energy $\epsilon, N$ is the number of mixture components, $\sigma_{k}(\epsilon)$ is the elastic cross section of the k-th component, $n_{k}$ is its number density and $\left(\sigma_{k}\right)_{i}(\epsilon)$ is the cross section for its $\mathrm{i}$-th inelastic process. The gas cross sections are extracted from the MAGBOLTZ program [16], while the electron energy and position are updated according to the classical dynamics of collision in an electric field. The interactions taken into account are : momentum transfer, excitation of electronic levels, excitation of vibrational levels, molecule dissociation, ionization, attachment.

In our scheme the photocathode and the anode lie in the $x y$ plane, at $z=0$ $\mathrm{mm}$ and $z=3.2 \mathrm{~mm}$ respectively, while the electric field is parallel to the $z$ axis. In our simplified modei, a photoelectron is considered backscattered, and thus not detected, when its $z$ coordinate becomes negative and is detected when it reaches the anode $(z \geq 3.2 \mathrm{~mm})$ or ionizes a gas molecule, since the chance that both the primary photoelectron and secondary electron are diffused back is negligible. Each backscattered electron is assumed to be absorbed at the photocathode surface and interactions with adsorbed gas molecules are neglected. For sake of simplicity the elastic scattering is considered isotropic, the avalanche multiplication is stopped at the first ionization and no secondary processes are taken into account.

At each set of experimental conditions, 1000 photoelectrons are generated and the percentage of those detected is to be compared directly to the ratio of gas to vacuum measured photocurrents.

\section{Simulation results and discussion.}

The results of Monte Carlo simulations for some of the investigated mixtures are shown in figs. 2,3 and 4 together with the respective measured gas-to-vacuum photocurrent ratios. The agreement between simulations and measurements results is satisfactory and the behaviour of the different gas mixtures is fairly well reproduced. As a consequence, we may attribute the major part of the reduction of photoelectron collection efficiency, observed in the investigated gas mixtures, to the backscattering phenomenon, which is the only one considered in our simplified model of electron transport.

The fractional energy transfer in an elastic collision is given by the well known relation $[15]$ :

$$
\frac{\Delta \epsilon}{c}=\frac{2 m M}{(m+M)^{2}}(1-\cos (\theta))
$$

where $m$ and $M$ are respectively the electron and gas molecule masses and $\theta$ is the scattering angle referred to the electron trajectory just before the impact. Due to 
the large mass difference, the energy loss per collision is very small, resulting in a wide range of scattering angles and in a high probability that some photoelectrons, backscattered towards the photocathode, will not be detected. In inelastic collisions the electron direction of motion is only slightly perturbed.

Therefore, in the framework of this simple model, the photoelectron collection efficiency is determined primarily by the ratio of elastic and inelastic collisions: the larger is the rate of inelastic collisions the lower is the backscattering probability. In the charge multiplication region, above the ionization threshold of the gas, the collection efficiency is close to the vacuum value due to the opening of several inelastic channels. This is true in all gases. In charge collection mode, at low electric field, the photoelectron collection efficiency depends strongly on the particular gas mixture: Helium has its first excitation energy at $19.82 \mathrm{eV}$ and ionization threshold at $24.59 \mathrm{eV}$, so in He-based gas mixtures, inelastic collision frequencies, under charge collection, are much smaller than elastic ones (fig. 5) and the photoelectron backscattering reduces the collection efficiency to $40 \%$ of the vacuum value (fig. 2).

In hydrocarbon gases $\left(\mathrm{CH}_{4}\right.$ and $\left.\mathrm{i}-\mathrm{C}_{4} \mathrm{H}_{10}\right)$ the presence of rotational and vibrational levels determines higher inelastic collision frequencies even at very low electric fields (fig. 5) and the backscattering effect is almost suppressed, thus generating a photoelectron collection efficiency of about $90 \%$ of the vacuum value (fig. 3 ).

Ar-based mixtures show a better collection efficiency than the corresponding $\mathrm{He}-$ based mixtures (fig. 4). A possible reason can be the lower thresholds for excitation and ionization of $\operatorname{Ar}(11.55 \mathrm{eV}$ and $15.7 \mathrm{eV}$, respectively) as well as the higher percentage of quenchers added to Ar.

\section{Conclusions}

It has been confirmed that the photoelectron collection efficiency in gas media can be reduced, with respect to vacuum, by the elastic backscattering from the gas molecules, depending on the gas nature and the $E / p$ value at the photocathode surface. At low $E / p$ values there is a striking evidence of this effect in He-based gas mixtures due to the lack of inelastic channels for electron-molecule interactions. At higher $E / p$ values the opening of excitation and ionization channels reduces the backscattering effect and the photoemission yield is close to vacuum values. In $\mathrm{CH}_{4}$-based mixtures, where inelastic collisions are possible even at low photoelectron energies, the photoemission yield is practically independent of the field and reaches the vacuum values at low $E / p$ and at atmospheric pressure. Therefore $\mathrm{CH}_{4}$ and $\mathrm{CH}_{4} / \mathrm{i}-\mathrm{C}_{4} \mathrm{H}_{10}$ $(95 / 5)$ seem to be the best candidates for the operation of UV-imaging gaseous detectors with a photoelectron collection efficiency almost free of backscattering effects and an effective quantum efficiency (QE) close to the intrinsic photocathode QE. A further improvement of the collection efficiency can be obtained by choosing the geometry of the gaseous detector such that the electric field at the photocathode surface 
corresponds to a gas gain above 100 .

The behaviour of Ar-based gas mixtures, similar to that of pure hydrocarbons, has a direct consequence on the design of CsI-based wire chambers for Cherenkov photons imaging in RICH, especially in large systems where the safety regulations impose a reduction of the amount of flammable gas. However further investigations are needed in order to tune the type and the exact amount of quenchers to be added in order to reduce the well known background originating from the scintillation of Ar.

In this article we treated the photoemission from CsI into gas media. It should be noted that the results are more general and are applicable to the emission from any solid photoconvertor into gas.

This work was done within the CERN-RD26 research project. It was supported by the Basic Research Foundation of the Israel Academy of Sciences and Humanities, the US-Israel BSF, by the Israel Ministry of Science and Arts, by the Commission of the European Communities and by the Foundation Mordoh Mijan de Salonique. A. Breskin is the W.P. Reuther Professor of research in the peaceful uses of atomic energy.

\begin{tabular}{||c||c|c|c||}
\hline GAS MIXTURE & $E / p[\mathrm{~V} / \mathrm{cm}$ Torr $]$ & $\mathrm{R}_{p e}(\mathrm{RICH})$ & $\mathrm{R}_{i}(\mathrm{LAB})$ \\
\hline \hline $\mathrm{Ar} / \mathrm{i}-\mathrm{C}_{4} \mathrm{H}_{10}(80 / 20)$ & 2.6 & 0.73 & 0.79 \\
\hline $\mathrm{Ar} / \mathrm{CH}_{4}(80 / 20)$ & 2.72 & 0.78 & 0.9 \\
\hline $\mathrm{Ar} / \mathrm{CH}_{4}(50 / 50)$ & 3.25 & 0.9 & 0.98 \\
\hline $\mathrm{He} / \mathrm{i}-\mathrm{C}_{4} \mathrm{H}_{10}(80 / 20)$ & 2.24 & 0.55 & - \\
\hline $\mathrm{He} / \mathrm{i}-\mathrm{C}_{4} \mathrm{H}_{10}(95 / 5)$ & 2.3 & - & 0.43 \\
\hline
\end{tabular}

Table 1 The ratio $R_{p e}$ of the average number of photoelectrons per event, detected with $\mathrm{RICH}$ in a given gas mixture, to that in $\mathrm{CH}_{4}$, at 1 atm; the ratio $\mathrm{R}_{i}$ of the photocurrent induced by $185 \mathrm{~nm}$ photons in the laboratory measured in a given gas mixture to that measured in $\mathrm{CH}_{4}$. Also given is the reduced electric field at the photocathode surface, in the RICH measurements. 


\section{References}

[1] See relevant articles on (si photocathodes in the Proc. of the first Workshop on RICH, Bari, Italy [Nucl. Instrum. and Meth. A343 (1994)], and in the Proc. of the second Workshop on RICH, Uppsala, Sweden [to be published in Nucl. Instrum. and Meth.] and references there.n.

[2] A. Di Mauro et al., Development of a large area advanced fast RICH detector for particle identification at the LHC operated with heavy ions, Preprint CERN/DRDC 94-49, RD26 Status Report, 1994.

[3] F. Piuz, RICH with CsI photocathodes. Presented at the second workshop on RICH, Uppsala, Sweden. These proceedings.

[4] H. Berger et al., Nurl. Instrum. and Meth. A360 (1995) 411.

[5] F. Piuz et al., Review of the development of CsI photocathodes for application to large RICH detectors. Presented at the Wire Chamber Conference, Vienna, Feb. 1995. To be published in Nucl. Instrum. and Meth.

[6] L.B. Loeb, Basic processes of gaseous electronics, University of California Press, Berkley 1955, p.601.

[7] R.W.L. Thomas and W.R.L. Thomas, J. Phys. B2 (1969) 562.

[8] G. Charpak et al., IEEE Trans. Nucl. Sc. NS-30 (1983) 134.

[9] A. Breskin et al., Nucl. Instrum. and Meth. A344 (1994) 163.

[10] A Breskin at al., Field-dependent photoelectron extraction from CsI in different gases, Preprint WIS-95/4/Feb.-PH, to be published in Nucl. Instr. and Meth. A.

[11] T.H. Di Stefano and E.W. Spicer, Phys. Rev. B7 (1973) 1554.

[12] A. Akkerman et al.. J. Appl. Phys. 76 (1994) 4656.

[13] R. Shimizu and D. Ze-Jun, Rep. Prog. Phys. 55 (1982) ,487.

[14] H.R. Skullerud, J. Phys. D1 (1968) 1567.

[15] G.W. Fraser and E. Mathieson, Nur tr. anci Meth. A247 (1986) 544.

[16] S.F. Biagi, Nucl. Instr. and Meth. A283 (1989) 716. 


\section{Figure captions}

Fig.1 CsI relative photoemission yield (symbols), at $185 \mathrm{~nm}$, and gas gain (lines) as a function of $E / p$ at photocathode surface in a) $\mathrm{He} / \mathrm{CH}_{4}(95 / 5)$ b) $\mathrm{He} / \mathrm{i}-\mathrm{C}_{4} \mathrm{H}_{10}$ $(95 / 5)$ and c) $\mathrm{CH}_{4} / \mathrm{i}-\mathrm{C}_{4} \mathrm{H}_{10}(95 / 5)$. The experimental data points are normalized to the averaged last three points.

Fig. 2 The ratio of the photocurrenc from CsI, at $185 \mathrm{~nm}$, in $\mathrm{He} / \mathrm{CH}_{4}(95 / 5)$ and $\mathrm{He} / \mathrm{i}-\mathrm{C}_{4} \mathrm{H}_{10}(95 / 5)$ at 800 Torr, to that in vacuum (measured at the same absolute electric field) and the simulation results, as a function of $E / p$. Simulated data refer to the percentage of detected photoelectrons out of 1000 generated.

Fig.3 The ratio of the photocurrent from CsI, at $185 \mathrm{~nm}$, in $\mathrm{CH}_{4}$ and $\mathrm{CH}_{4} / \mathrm{i}-\mathrm{C}_{4} \mathrm{H}_{10}$ $(95 / 5)$ at 800 Torr to that in vacuum (measured at the same absolute electric field) and the simulation results, as a function of $E / p$. Simulated data refer to the percentage of detected photoelectrons out of 1000 generated.

Fig.4 The ratio of the photocurrent from CsI, at $185 \mathrm{~nm}$, in pure $\mathrm{Ar}$ and $\mathrm{Ar} / \mathrm{CH}_{4}$ $(50 / 50)$ at 800 Torr to that in vacuum (measured at the same absolute electric field) and the simulation results, as a function of $E / p$. Simulated data refer to the percentage of detected photoelectrons out of 1000 generated.

Fig.5 Collision frequencies in $\mathrm{He} / \mathrm{CH}_{4}(95 / 5)$ and $\mathrm{CH}_{4}$ at 800 Torr, calculated as a function of $E / p$ in the Monte Carlo program. 


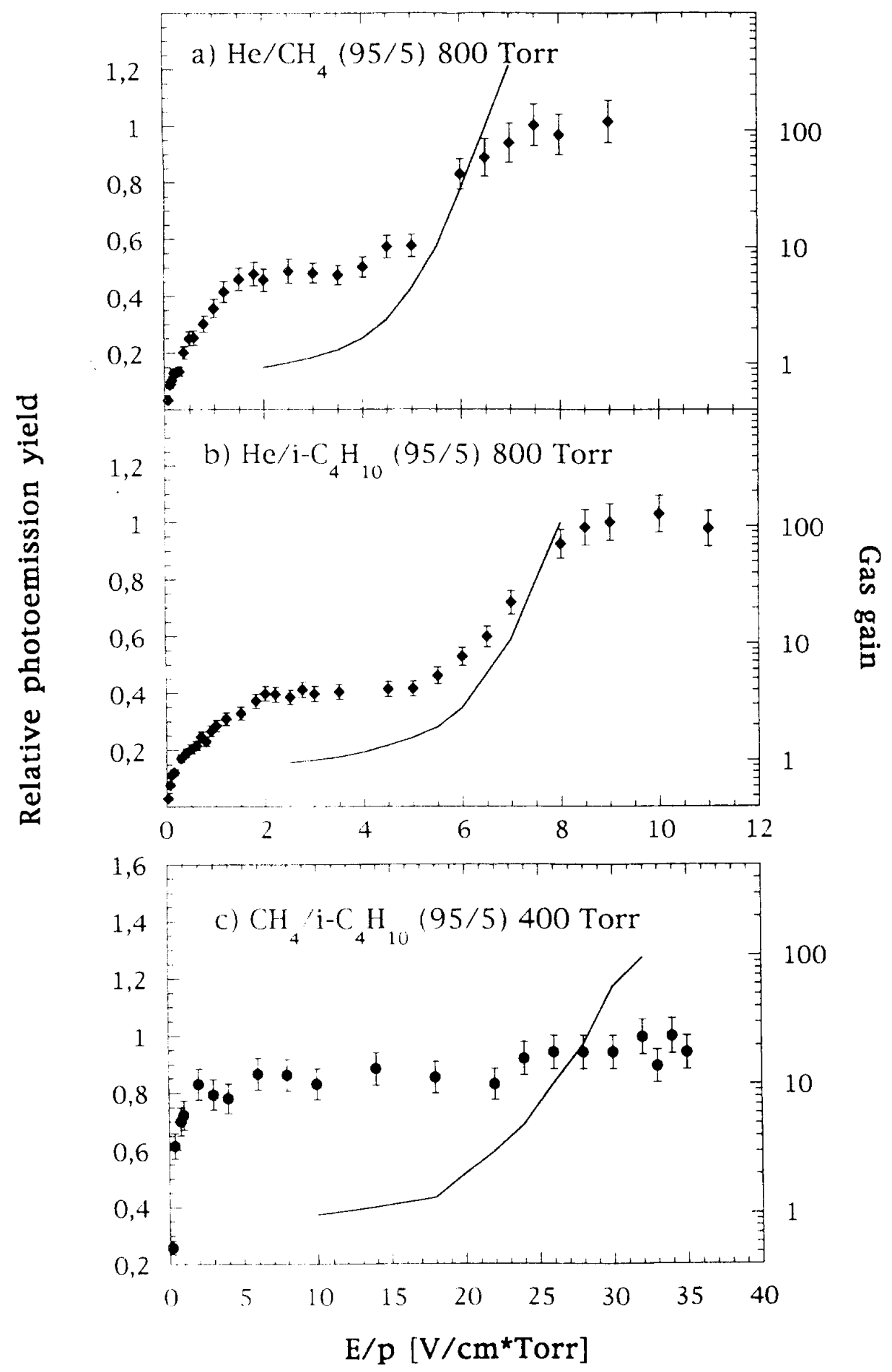

Figure 1 


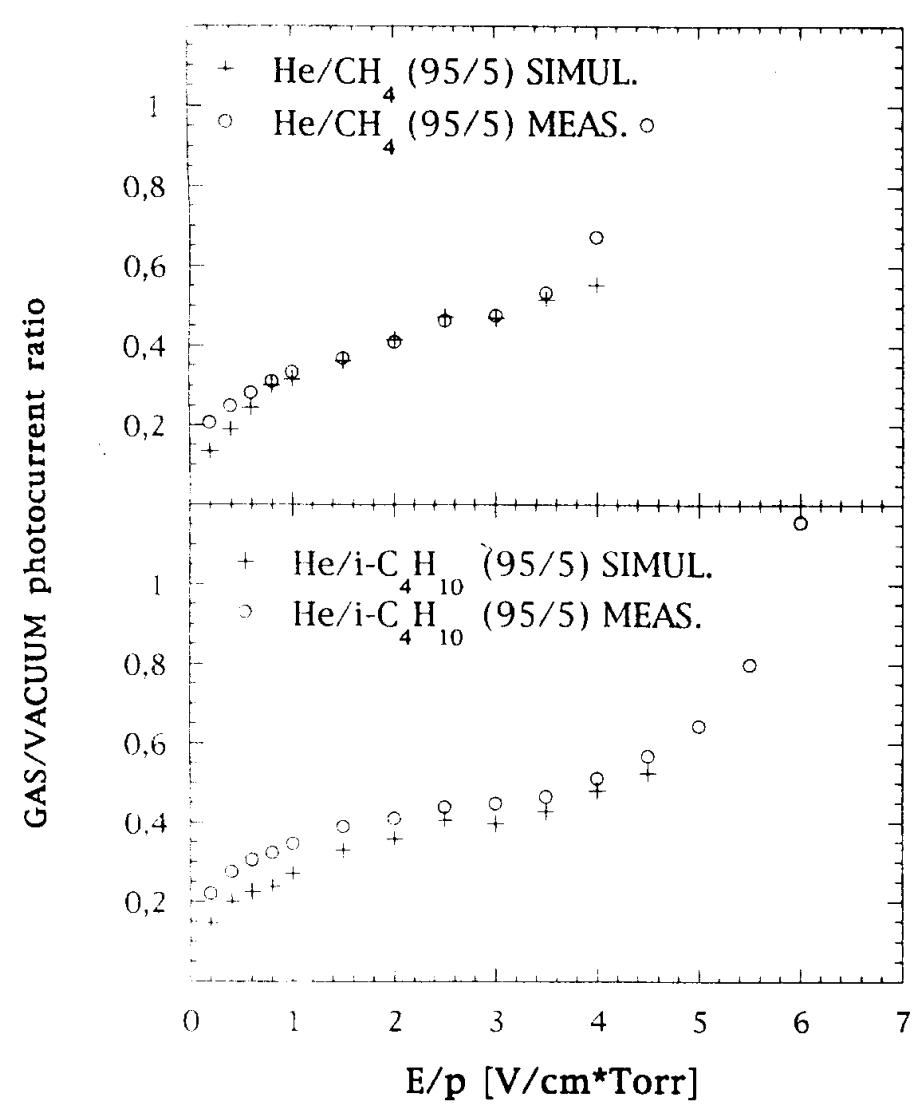

Figure 2

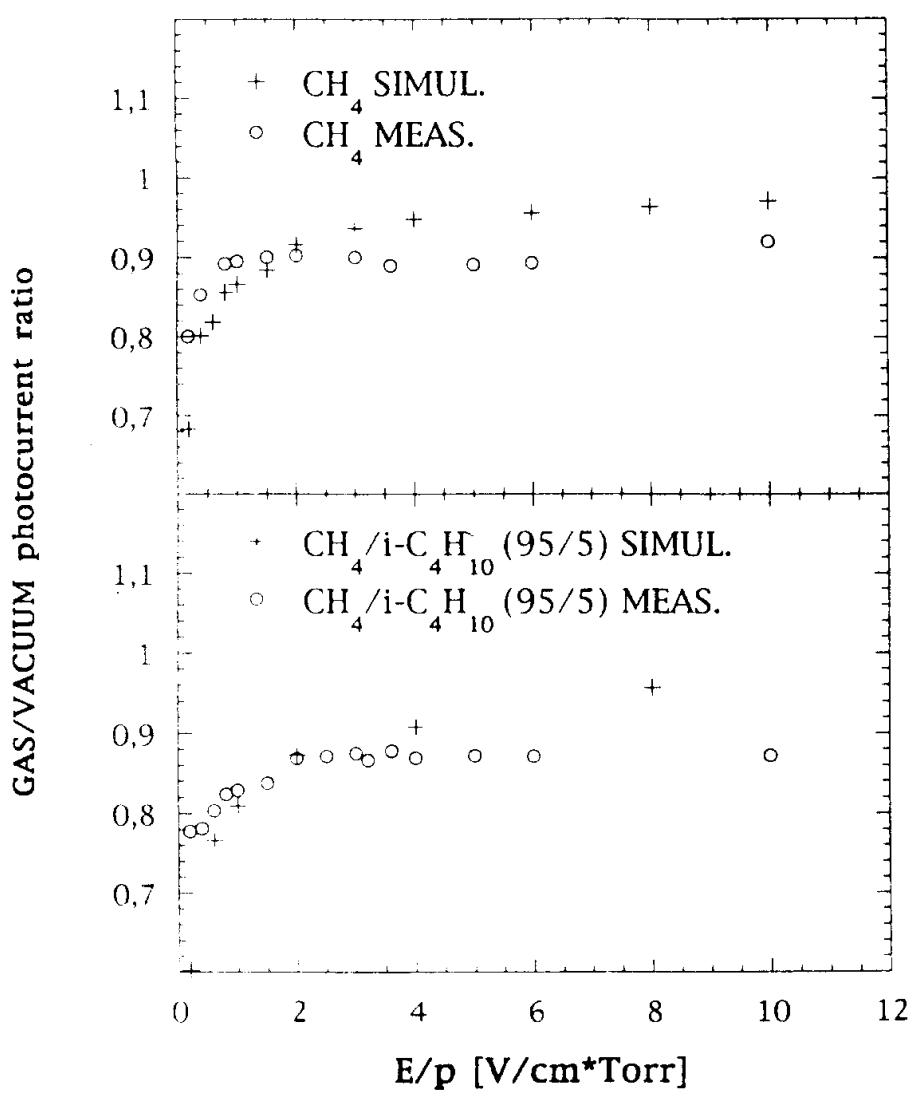

Figure 3 


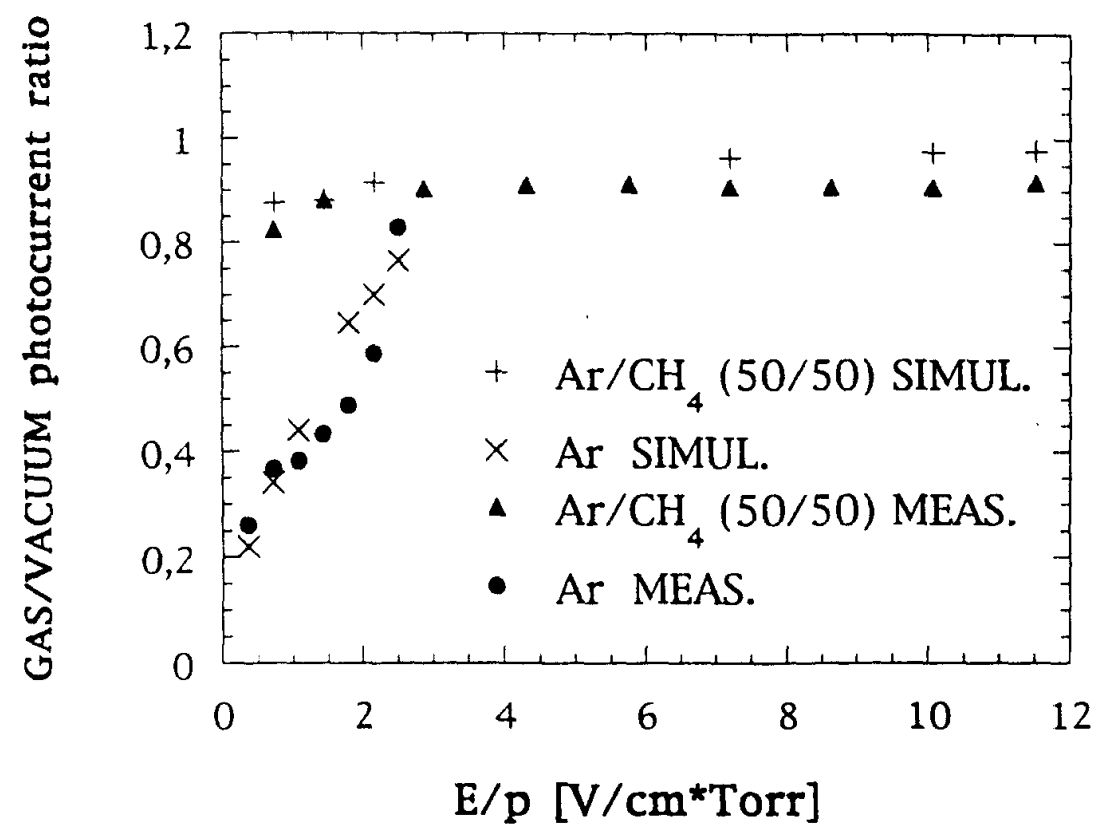

Figure 4

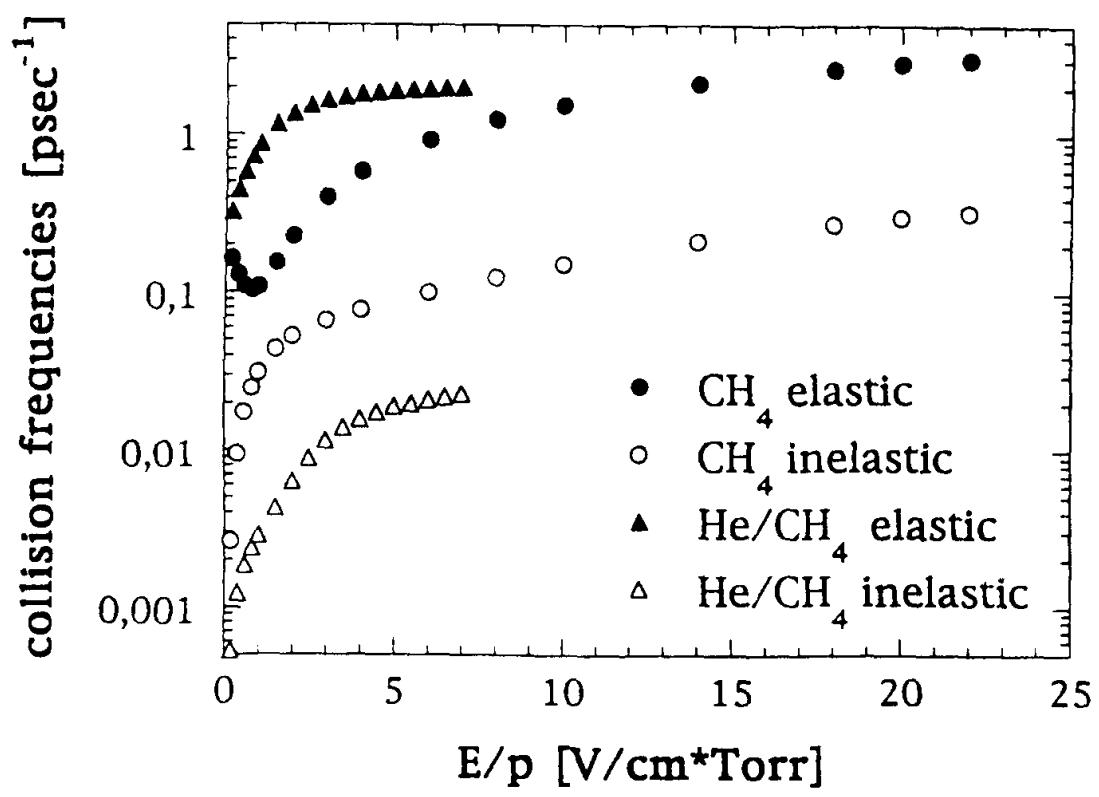

Figure 5. 
